\title{
A Differential Rotation Law for Stars and Fluid Planets
}

\author{
Joel Uriel Cisneros-Parra ${ }^{1,2,}$, Francisco Javier Martínez-Herrera ${ }^{2}$, Daniel Montalvo-Castro ${ }^{2}$ \\ ${ }^{1}$ Science Faculty, Autonomous University of San Luis Potosí, San Luis Potosí, México \\ ${ }^{2}$ Physics Institute, Autonomous University of San Luis Potosí, San Luis Potosí, México \\ Email address: \\ cisneros@galia.fcuaslp.mx (J. U. Cisneros-Parra), marherrera2011@hot.mail.com (F. J. Martínez-Herrera), \\ montalvo@ifísica.uaslp.mx (D. Montalvo-Castro) \\ ${ }^{*}$ Corresponding author
}

\section{To cite this article:}

Joel Uriel Cisneros-Parra, Francisco Javier Martínez-Herrera, Daniel Montalvo-Castro. A Differential Rotation Law for Stars and Fluid Planets. American Journal of Astronomy and Astrophysics. Vol. 8, No. 2, 2020, pp. 30-34. doi: 10.11648/j.ajaa.20200802.13

Received: March 31, 2020; Accepted: May 3, 2020; Published: May 15, 2020

\begin{abstract}
We derive, supported on a generalization of Bernoulli's equation, a law of rotation for any axial-symmetric, self-gravitating fluid mass. For a homogeneous mass, the law depends solely on the derivative of the potential with respect to the distance to the rotation axis, implying generally differential rotation, the Maclaurin spheroids representing the only case of solid-body rotation. We turn then to a heterogeneous mass consisting of any number $l$ of concentric layers, each of constant density, finding that the angular velocity profile of a given layer depends on that of the layer immediately above it. Finally, we let $l$ tend to infinity to convert our model into continuous mass distribution, the result being a certain rotation profile for the surface, and law of differential rotation change at its interior. To support the fundamentals of our approach, we write the potential integrals for the three mass distributions. The aim of a continuous distribution is that it may facilitate a comparison---to be carried out in a future paper---between our results and those of other researchers who employ structure equations. We point out that the distribution of angular velocity is a consequence of the equilibrium, rather than being imposed ad initio. The law was used in a past paper to construct a Jupiter multi-layer model adopting the spheroidal (a distorted spheroid) shape for each of the layers, taking as reference the gravitational data surveyed by the Juno mission. The procedure used here is not restricted to axial-symmetric cases.
\end{abstract}

Keywords: Gravitation, Hydrodynamics, Planets and Satellites, Stars, Rotation

\section{Introduction}

In the current paper, we derive, consistent with Euler's steady-state equations of fluid motion (zero viscosity), a law of rotation valid for any self-gravitating, axial-symmetric fluid mass. The fundamentals of our formulation were previously discussed [1,2], and only its main aspects will be recalled here. In essence, the law states that the angular velocity at the body's surface is proportional to the derivative of the potential with respect to $R^{2}$, where $R$ is the distance from the rotation axis. Since the Maclaurin spheroid is the unique case in which the potential at the surface is a linear function of $R^{2}$, it must rotate as a solid-body; in other cases, the angular velocity necessarily varies on the surface, that is, it is of a differential type. This outcome convinced us that, given the mass' shape, there is no freedom in choosing the angular velocity. Conversely, given the angular velocity profile, is it possible to deduce the surface shape? For example, it is known $\{3\}$ that the Sun rotates differentially at a rate given by the approximate formula

$$
\omega=2.87 \times 10^{-6}\left(1+0.12 \cos ^{2} \vartheta-0.17 \cos ^{4} \vartheta\right) \mathrm{rad} \mathrm{s}^{-1}
$$

where $\vartheta$ is the colatitude measured from the rotation axis, and $\cos \vartheta=z / \sqrt{R^{2}+z^{2}}$ (in terms of the variables we will use here). Since the surface potential (its derivative), is related to $\omega^{2}$, the expectation is that the Sun's rotation would determine its potential. However, the object's shape is not implicit in equation (1), that is, the function $z=f(r)$ is not known, and without this information, the surface potential cannot be calculated. Since for the time being applications are not intended, the solar rotation will be deferred to future work.

Recently [4], we built a model for Jupiter made out of a number $l$ of concentric layers, each of constant density., and each rotating with its distribution of angular velocity. Given 
that Jupiter's shape is unknown, we assumed that each layer is a distorted spheroid (a spheroidal, following Jeans [5]), with a fourth-order distortion term in the $z$-direction (the rotation axis). The resulting figure $(l=15$, say) rotates with an equatorial period of about $10 \mathrm{~h}$, considerably faster than the Sun (about 25 days); this means that Jupiter must be more distorted than the Sun. The theoretical results for Jupiter can be expressed by the approximate formula:

$$
\omega=1.70 \times 10^{-5}\left(1+0.66 \cos ^{2} \vartheta-0.02 \cos ^{4} \vartheta\right) \mathrm{rad} \mathrm{s}^{-1}
$$

In the first few sections of the current paper, we recapitulate and precise our earlier results. To reach our Jupiter model, we started with a homogeneous spheroidal mass, which was next invested with a concentric---but otherwise free from any relation between their respective semi-axes---spheroidal layer; we refer to the two layers of this model as the core and the envelope. Usually, the core is denser than the envelope, but the opposite can also be possible; furthermore, the prolate form for the core is possible for low relative densities. Next, the Jupiter model was made to consist of any number $l$ of concentric layers, which improved the results of $l=2$. In the current paper, we revise, regardless of the shape, the multi-layer distribution, and we extend our results to a continuous distribution attained by letting $l$ tend to infinity. The potential integrals are written explicitly. The aim of studying a continuous distribution is that it may facilitate a comparison (to be carried out in future work) between our results and those by other researches who employ structure equations. We remark that in our approach the rotation profiles are a consequence of the theory, rather than being pre-imposed.

\section{The Law of Rotation}

\subsection{The Homogeneous Mass}

In a past paper [1], we worked out the case of homogeneous fluid motion in steady-state, of which only the more relevant aspects will be reproduced here. The basis of our procedure rests on Euler's equations:

$$
\frac{\partial v}{\partial t}+(v \cdot \nabla) v=\nabla V-\frac{1}{\rho} \nabla p, \frac{\partial \rho}{\partial t}+\nabla \cdot \rho v=0
$$

where $v$ is the velocity field, $V$ the gravitational potential, $p$ the pressure, and $\rho$ the density at each point of the fluid at time $t$. In the special case of a stationary state, which will exclusively be considered here, all variables depend on the position but are independent of time. Hence, equations (3) become

$$
(v \cdot \nabla) v=\nabla V-\frac{1}{\rho} \nabla p, \nabla \cdot \rho v=0
$$

These expressions will be applied first to a fluid of constant density, independently if it is incompressible or not, so that equations (4) become

$$
(v \cdot \nabla) v=\nabla V-\frac{1}{\rho} \nabla p, \nabla \cdot v=0
$$

Using a known vector identity, the left-hand side of the first of equations (5) becomes

$$
\nabla\left(\frac{1}{2} v^{2}-V+\frac{1}{\rho} p\right)=v \times(\nabla \times v)
$$

For a streamline (tangent to $v$ ) is valid the relation (Bernoulli's equation)

$$
\frac{1}{2} v^{2}-V+\frac{1}{\rho} p=k
$$

$k$ being a constant. In general, $k$ changes from one streamline to another: $k=k(x, y, z)$. Therefore, according to equation (4), $k$ must satisfy the relation

$$
\nabla k=v \times(\nabla \times v)
$$

for any velocity field. From equation (8), it follows that the special case for which $k$ is constant everywhere is the irrotational flow. In what follows, the shape of the mass will be restricted to have axial symmetry $\left(f\left(x^{2}+y^{2}, z^{2}\right)=0\right)$. Additionally, it will be assumed that the motion is of a rotatory kind, so that the velocity field is given by

$$
v=(-\omega y, \omega x, 0)
$$

where $\omega$ is the usual non-constant angular velocity, and the parenthesis is a vector with components $-\omega y, \omega x$, and 0 along the cartesian axes. The continuity equation (second of equations (3)) puts a restriction on the velocity field (9), expressed as

$$
\frac{\partial \omega}{\partial x^{2}}=\frac{\partial \omega}{\partial y^{2}}
$$

This means that, generally, the angular velocity must be a function of the kind

$$
\omega=\omega\left(x^{2}+y^{2}, z^{2}\right)
$$

where it is assumed that the velocity field is symmetric regarding the $z$-axis. In cylindrical coordinates $(R, \varphi, z)$ the problem is independent of $\varphi$, and the velocity field (tangent to circles) has only a $\varphi$-component:

$$
v=(0, \omega R, 0)
$$

The parenthesis being a vector with components $0, \omega R, 0$ along to the unit vectors $i_{R}, i_{\varphi}, i_{z}$ so that $v$ has the non-zero component $v_{\varphi}=\omega R$ only. From equation (8), we have

$$
\frac{\partial k}{\partial R^{2}}=\omega\left(R^{2} \frac{\partial \omega}{\partial R^{2}}+\omega\right), \frac{\partial k}{\partial z^{2}}=\omega R^{2} \frac{\partial \omega}{\partial z^{2}}
$$

The general solution of equations (13) can be found as was done in [1], namely with the variable change

$$
\omega=\sqrt{\frac{2 \Omega}{R^{2}}}
$$

So that equations (13) become

$$
\frac{\partial k}{\partial R^{2}}=\frac{\partial \Omega}{\partial R^{2}}+\frac{1}{R^{2}} \Omega, \frac{\partial k}{\partial z^{2}}=\frac{\partial \Omega}{\partial z^{2}}
$$


From the second of equations (15), we deduce

$$
k\left(R^{2}, z^{2}\right)=\Omega\left(R^{2}, z^{2}\right)+f\left(R^{2}\right)
$$

where $f$ is an arbitrary function. Thence, the first of equations (15) implies that

$$
\Omega\left(R^{2}, z^{2}\right)=R^{2} f^{\prime}\left(R^{2}\right), \text { or } \omega^{2}=2 f^{\prime}\left(R^{2}\right)
$$

i.e. the angular velocity can be at most a function of $R$ only, and likewise $k$ :

$$
k=\frac{1}{2} R^{2} \omega^{2}+f\left(R^{2}\right), \omega^{2}=2 f^{\prime}\left(R^{2}\right)
$$

Since the angular velocity does not depend on $z$, it is constant on cylinders of radius $R$, a well-known result established long ago ([6], pp. 176-178)

Substituting $k$ of equation (18) in Bernoulli's equation (7), we obtain, after some elementary algebra

$$
-f\left(R^{2}\right)-V\left(R^{2}, z^{2}\right)+\frac{1}{\rho} p=0
$$

Since function $f$ depends on $R$ but not on $z$, to evaluate it we require only the boundary surface of the figure, where $p=0$ :

$$
f\left(R^{2}\right)=-V\left(R^{2}, z^{2}\right)
$$

where $z$ is a function of $R$ for a mass with cylindrical symmetry. Equation (20) allows to determine the unknown function $f$ $(=-V)$, and with equation (18), $\omega$ is established:

$$
\omega^{2}=-2 \frac{d V}{d R^{2}}
$$

Equation (21) is the general law of rotation for an axial-symmetric self-gravitating homogeneous fluid.

\subsection{The Multi-layer Mass}

In this section, the configuration of our model consists of any number $l$ of concentric layers, each of constant density $\rho_{i}(i=1, \ldots, l), \rho_{1}$ is the density of the outermost layer. For any shell $i$, the equilibrium equation (19) holds

$$
\frac{1}{\rho_{i}} p_{i}=f_{i}\left(R^{2}\right)+V_{i}\left(R^{2}, z^{2}\right)
$$

where $V_{i}\left(R^{2}, z^{2}\right)$ is the total potential at the point $\left(R^{2}, z^{2}\right)$ of the body, $f_{i}$ an unknown function, and $p_{i}$ the pressure at the same point. The $l$ unknown functions are established employing the boundary conditions at several interfaces:

$$
\text { Surfaces } S_{1}: p_{1}=0, S_{2}: p_{1}=p_{2}, S_{i}: p_{i}=p_{i-1} \text {. }
$$

No transfer of mass at the interfaces is assumed [7]. The surfaces $S_{i}$ are concentric but otherwise independent of one another. Any surface equation is of type $g(R, z)=0$, so that, on solving for $z$ we get $z=h(R)$, and all quantities in equation (22) depend only on $R$. Therefore, according to equation (22), and knowing that the potential is continuous, from the boundary conditions there result

$$
\frac{1}{\rho_{1}} p_{1}=0=f_{1}\left(R^{2}\right)+V_{1}\left(R^{2}, z^{2}\right), \text { or } f_{1}=-V_{1},
$$

$$
p_{i}=p_{i-1}:\left(\rho_{i}-\rho_{i-1}\right) V_{i}+\rho_{i} f_{i}-\rho_{i-1} f_{i-1}=0
$$

Or

$$
f_{i}=-\frac{\delta \rho_{i}}{\rho_{i}} V_{i}+\frac{\rho_{i-1}}{\rho_{i}} f_{i-1}
$$

where $\delta \rho_{i}=\rho_{i}-\rho_{i-1}, \delta \rho_{1}=\rho_{1}$

Taking the derivative of equation (23) with respect to $R^{2}$ and using relation (21), we obtain for the angular velocity

$$
\omega_{i}^{2}=-2 \frac{\delta \rho_{i}}{\rho_{i}} \frac{d V_{i}}{d R^{2}}+\frac{\rho_{i-1}}{\rho_{i}} \omega_{i-1}^{2}
$$

Equation (24) is the rotation law for the multi-layer mass. In general, the gravitational potential is not a linear function of $R^{2}$ at each interface point (except for a Maclaurin spheroid), so that every layer rotates with its particular differential angular velocity.

\subsection{The Continuous Mass}

We will now direct our attention to the more general case where the density varies continuously from the surface to the center. This is achieved by letting the number of layers $l$ tend to infinity while their widths tend to zero; also, $\delta \rho_{i}$ tends to zero. Let us call $\omega_{i}^{2}=\omega^{2}=\omega_{i-1}^{2}+d \omega^{2}$. Taking the limit of equation (24), we obtain

$$
\frac{d \omega^{2}}{d \rho}=-\frac{2}{\rho} \frac{d V}{d R^{2}}
$$

In this procedure, the mass is tacitly divided into isodensity surfaces, each one rotating differentially at a rate given by equation (25); we remark that the surfaces are axially symmetric. Substituting in the gravitational potential $V$ an equation of the type $z=g\left(R^{2}\right), V$ becomes a function of $R^{2}$. Additionally, a reasonable boundary condition is to suppose that the surface of the mass is isobaric. Then the pressure $p_{S}$ is constant and can be small or zero. Let the density on the surface be $\rho_{S}$. Hence, according to equation (16), we have

$$
\frac{1}{\rho_{S}} p_{S}=f_{S}\left(R^{2}\right)+V_{S}\left(R^{2}, z^{2}\right)
$$

where $f_{S}\left(R^{2}\right)$ and $V_{S}\left(R^{2}, z^{2}\right)$ are functions referred to points on the surface. Since the shape of the mass is of the form $z=g\left(R^{2}\right)$, the potential becomes a function of $R^{2}$, and therefore the angular velocity $\omega_{S}^{2}$ for the surface $S$ is established (see also equations (18)):

$$
\omega_{S}^{2}=-\frac{d V_{S}}{d R^{2}}
$$

Equation (27) is the starting point for, in principle, determining the angular velocity at any internal point, and equation (25) allows to fix, step, by step, the changes in $\omega^{2}$.

\section{The Potential}

\subsection{The Multi-layer Potential}

As in section 2.3, we consider here a body consisting of $l$ layers, the outermost being limited by the surfaces $S_{1}$ (upper) and $\mathrm{S}_{2}$ (lower); we call $\Delta \tau_{1}$ and $\rho_{1}$ its volume and density, respectively. The volume enclosed by $S_{1}$ is designated by $\tau_{1}$ 
and corresponds to the whole body's volume $\tau$. In genera

1 , the $i$ layer is limited by the $\mathrm{S}_{\mathrm{i}}$ and $\mathrm{S}_{\mathrm{i}+1}$ surfaces enclosing the volumes $\tau_{i}$ and $\tau_{i+1}$ respectively. The volume of the $i$ layer will be denoted by $\Delta \tau_{i}$. Let $r$ be a point on the surface $S_{m}$. Hence, the total gravitational potential is given by the well-known expression

$$
\begin{aligned}
& V(r)=G \int_{\tau^{\prime}} \frac{\rho\left(r^{\prime}\right)}{\left|r-r^{\prime}\right|} d \tau^{\prime} \\
& \quad V(r)=G\left(\rho_{1} \int_{\tau_{1}} \frac{d \tau^{\prime}}{\left|r-r^{\prime}\right|}+\left(\rho_{2}-\rho_{1}\right) \int_{\tau_{2}} \frac{d \tau^{\prime}}{\left|r-r^{\prime}\right|}+\cdots\right)=G \sum_{i=1}^{l} \delta \rho_{i} \int_{\tau_{i}} \frac{d \tau^{\prime}}{\left|r-r^{\prime}\right|}
\end{aligned}
$$

where $\delta \rho_{i}=\rho_{i+1}-\rho_{i}, \delta \rho_{1}=\rho_{1}$

The mass enclosed by the surface $S_{m}$ contributes to the total potential at the point $r$ as an external potential; the mass above $S_{m}$ contributes as an internal potential. Therefore, equation (29) is equivalent to

$$
V(r)=G \sum_{i=1}^{m} \delta \rho_{i} \int_{\tau_{i}} \frac{\rho(r \prime)}{\left|r-r^{\prime}\right|} d \tau^{\prime}+G \sum_{i=m+1}^{l} \delta \rho_{i} \int_{\tau_{i}} \frac{\rho\left(r^{\prime}\right)}{\left|r-r^{\prime}\right|} d \tau^{\prime}
$$

The first sum is the interior part of the potential, i.e., the point $r$ is an inner point of the distribution. The second sum is the outer part of the potential ( $\mathrm{r}$ lies outside the distribution).

\subsection{The Continuous Case}

For a continuous distribution of mass, we take in equation (30) the limit for an infinite number of layers of infinitely small thickness $\left(\delta \rho_{i} \rightarrow 0, \delta \rho_{1} \neq 0\right)$ resulting in

$$
V(r)=G \rho_{1} \int_{\tau} \frac{\rho\left(r^{\prime}\right)}{\left|r-r^{\prime}\right|} d \tau^{\prime}+G \int_{\rho_{1}}^{\rho} \int_{\tau^{\prime} \text { in }} \frac{\rho\left(r^{\prime}\right)}{\left|r-r^{\prime}\right|} d \tau^{\prime}+G \int_{\rho}^{\rho_{c}} \int_{\tau^{\prime} e x} \frac{\rho\left(r^{\prime}\right)}{\left|r-r^{\prime}\right|} d \tau^{\prime}
$$

Notice that, for reaching this result, the body is subdivided into (axial-symmetric) surfaces of constant density. The $\rho$ integrals are evaluated by computing first the potential over the volume ( $\tau_{\text {in }}^{\prime}$ or $\tau_{\text {ex }}^{\text {ex }}$ ) limited by a surface $\rho^{\prime}=$ const., for each $\rho^{\prime}$ of the interval; here, $\rho_{c}$ is the density at the center, and $\rho_{1}$ is the (constant) density at the body's surface. For stars and liquid planets, the density at the "boundary" is small and can be taken as zero. In equation (27), the first term is kept for reasons of generality, the potential is for a point $r$ at the surface $\rho=$ const.. Obviously, this procedure can be applied to any integral of type (28), such as

$$
\int_{\tau} \rho\left(r^{\prime}\right) F\left(r^{\prime}\right) d \tau^{\prime}=\rho_{S} \int_{\tau} F\left(r^{\prime}\right) d \tau^{\prime}+\int_{\rho_{S}}^{\rho_{c}} d \rho^{\prime} \int_{\tau^{\prime}} F\left(r^{\prime}\right) d \tau^{\prime}
$$

The integrands in equations (31) and (32) of the integrals in $\tau^{\prime}$ implicitly depend on $\rho^{\prime}$, and they must be calculated at each point of the corresponding isodensity surface. In particular, if the surfaces are established by a set of parameters, where any of them must be generally considered as dependent of $\rho$.

\section{Conclusion}

In the way that conduced to the rotation law

$$
\omega^{2}=-2 \frac{d V}{d R^{2}}
$$

we took two natural steps: the well-known facts that the angular velocity is constant on cylinders and that the motion has an effective potential (equation (19)):

$$
V_{\mathrm{eff}}=V\left(R^{2}, z^{2}\right)+f\left(R^{2}\right)
$$

which was not called by its name, because is not important for our study. It is equivalent to the commonly used potential ([6], p. 68)

$$
\Phi=\mathrm{V}\left(\mathrm{R}^{2}, \mathrm{z}^{2}\right)+\int^{R} \omega^{2}\left(R^{\prime}\right) R^{\prime} d R^{\prime} .
$$

Certainly, remembering that $\omega^{2}=2 f^{\prime}\left(R^{2}\right)$ (equation (21)) and integrating this respect to $R$, one gets $\Phi$. The law $\Phi=$ const. and our rotation law are equivalent. Nonetheless, ours has the advantage of being expressed in a differential form, since one is more familiarized with such representations. Equation $\omega^{2}=$ $-d V / d R^{2}$ shows clearly the relation between the rotation profile and the body's shape (that fixes $V$ ): knowing the shape and therefore, the potential, the rotation profile is established; one cannot use this profile for a distinct form because its potential function will be different. The integral representation of the effective potential can eventually induce confusion when using it in an application. For example, choosing a constant angular velocity (a common case, for example [9]) in $\Phi$ and intending to find a figure that is not a spheroid would be impossible. However, the differential relation teaches that only the spheroid has $\omega=$ const because the potential is a linear function of $R^{2}$ on its surface (Maclaurin spheroid

In our model the pressure is continuous at each point of a shell surface and, in general, it is not constant on the whole surface, because of differential rotation. Hence, isodensity surfaces are not isobaric. An assumption of a relation between $p$ and $\rho$ cannot be done here (barotropic relation, like in Ostriker and Mark [14]). In studying small oscillations of differentially rotating gas masses, Tassoul assumes a relationship between the 
variations of $p$ and $\rho$, but not for the variables themselves [15].

Notice that our procedure is not restricted to the rotation state of axial-symmetric mass distributions. This is because it is sustained on the generalized form of Bernoulli's equation (equations (7) and (8))

$$
\frac{1}{2} v^{2}-V+\frac{1}{\rho} p=k
$$

with $k$ given by

$$
\nabla k=v \times(\nabla \times v)
$$

and these equations are not restricted to any particular (symmetric) motion. For instance, if they are applied to the solid-body rotation of an ellipsoid (three unequal axes, Jacobi ellipsoid $[10,11])$ or the static one with internal currents of uniform vorticity (Dedekind ellipsoid [12, 13]), the known results are reproduced easily ([1]). We aim to study cases of non-axial symmetric motions in the near future.

\section{References}

[1] Cisneros, J. U., F. J. Martínez, \& D. Montalvo, RMx, AA, 2016, 52,375 .

[2] Cisneros, J. U., F. J. Martínez, \& D. Montalvo, 2017, ApJ, 848, 109.

[3] Kitchatinov, L. L., 2005, Physics-Uspekhi, 48 (5), 449.
[4] Cisneros, J. U., F. J. Martínez, \& D. Montalvo, American Journal of Astronomy and Astrophysics. Vol. 8, No. 1, 2020, pp. 8-14.

[5] Jeans, J. H. 1919, Phil Trans. R. Soc., (Cambridge, England Cambridge University Press).

[6] Poincare, H., Theorie des Turbillons, (1893), Gauthier-Villars, Paris, 212.

[7] Landau, L. $\backslash$ D. $\backslash \backslash \&$ Lifshitz E. $\backslash$ M., 1987, Course of Theoretical Physics: Fluid Mechanics, New York: Pergamon Press.

[8] Tassoul, J. L., Stellar Rotation, (2000), Cambridge University Press, 256.

[9] Hubbard, W. B., 2012, ApJL, 756: L15.

[10] Jacobi, C. G. J., Uber die Figur des Gleichgewichts, Poggendorff Annallen der Physik und Chemie, Vol. 33, 1834, pp. 229-238; reprinted in Gesammelte Werke, Vol. 2, pp. 17-72, G. Reimer, Berlin, 1882.

[11] Lyttleton, R. A. 1953, The Stability of Rotating LiquidMasses (Cambridge: Cambridge University Press).

[12] Chandrasekhar, S. 1969 Ellipsoidal Figures Of Equilibrium (Yale: University Press)

[13] Dedekind, R., Zusatz zu der vorstehenden Abhandlung, J. Reine Angew. Math., Vol. 58, 1860.

[14] Ostriker, J. P., J. W-K. Mark, 1968, ApJ, 151.

[15] Tassoul, J. L., J. P. Ostriker ApJ, 1968, 154, 613. 